\title{
GENERALIZED CONTRACTION PRINCIPLE
}

\author{
DIPAK CHATTERJEE \\ St. Xavier's College \\ 30, Park Street \\ Calcutta-700016, INDIA
}

(Received March 23, 1982 and in revised form August 3, 1982)

ABSTRACT. Fixed point theorems are proved for contraction maps on M-convex spaces.

KEY WORDS AND PHRASES. Fixed Point Theorems, Contraction principle, M-convex spaces. 1980 MATHEMATICS SUBJECT CLASSIFICATION CODES.

\section{INTRODUCTION.}

Since Banach published his pioneering work on fixed points, various extensions in multidirections have been achieved by Brouwer, Kakutani, Browder, Edelstein, Kannan, Soardi, Rakotch, Reich, Chi Song Wong, Meir and Keeler, Hardy and Rogers, Chatterjee and many others. The ones relevant to our work are due to Edelstein [1], Kannan [2], Reich [3], Wong [4] and Hardy and Rogers [5]. In fact, Kannan [2] proved that, if $\mathrm{K}$ is a weakly compact convex subset of a reflexive Banach space and if for every closed convex subset $\mathrm{H}$ with $\mathrm{T}(\mathrm{H}) \subseteq \mathrm{H}, \delta(\mathrm{H})>0$, inf || $\mathrm{y}-\mathrm{Ty} \|<\delta(\mathrm{H})$ and if $\mathrm{T}$ is a self-map of $\mathrm{K}$ such that for every pair of points $\mathrm{x}, \mathrm{y} \in \mathrm{H},\left\|\mathrm{Tx}-\mathrm{Ty}_{\mathrm{y}}\right\| \leq \frac{1}{2}\{|| x-\mathrm{Tx}||+|| \mathrm{y}-\mathrm{Ty}||\}$, then $\mathrm{T}$ has a fixed point. Reich proved that, if $\mathrm{K}$ is a weakly compact convex subset of a Banach space and $T$ is a self-map of $K$ such that there exist $\left\{a_{i}\right\}_{i=1}^{5}, a_{i} \geq 0$, $\sum a_{i}=1$, and for every pair of points $x, y \in k,\|T x-T y\| \leq a_{1}|| x-y||+a_{2}|| x-T \|+$ $a_{3}|| y-T y||$, then $T$ has a fixed point. Hardy and Rogers extended this result to metric spaces as follows. If $(X, d)$ is a complete metric space and $T$ is a self-map of $X$ such that there exists $\left\{a_{i}\right\}_{i=1}^{5}, a_{i} \geq 0, \sum a_{i}<1$ and $d(T x, T y) \leq a_{1} d(x, y)+a_{2} d(x, T x)+$ $a_{3} d(y, T y)+a_{4} D(x, T y)+a_{5} d(y, T x)$ for every pair of points $x, y \in X$, then $T$ has $a$ fixed point. The conditions that $X$ is compact and $T$ is continuous are sufficient if the condition $\sum a_{i}<1$ is changed to $\sum a_{i}=1$. Wong generalized the results of Edelstein, 
Kannan and Reich by way of proving that, if $X$ is a convex subset of a normed linear space and $T$ is a self-map of $X$ such that there exist $\left\{a_{i}\right\}_{i=1}^{5}, a_{i} \geq 0, \sum_{i}=1$ and for all $\mathrm{x}, \mathrm{y} \in \mathrm{x},|| \mathrm{Tx}-\mathrm{Ty}|| \leq \mathrm{a}_{1}|| \mathrm{x}-\mathrm{y}\left\|+\mathrm{a}_{2}|| \mathrm{x}-\mathrm{Tx}||+\mathrm{a}_{3}|| \mathrm{y}-\mathrm{Ty}||+\mathrm{a}_{4}|| \mathrm{x}-\mathrm{Ty}\right\|+$ $a_{5}|| y-T x||$, then $T$ has a fixed point and this point is the limit of $x_{n}$ where $x_{n}=(1-t) x_{n-1}+t T x_{n-1}, t \in(0,1)$. In fact, Edelstein's result follows from Reich if $a_{2}=a_{3}=a_{4}=a_{5}=0$; Kannan's result follows by putting $a_{1}=0, a_{2}=a_{3}=1 / 2$; Reich's result can be deduced by substituting $a_{4}=a_{5}=0$. Ours is a further generalization of all the above results from two angles, one being the liberation of the selection conditions on $a_{1}, a_{2}, a_{3}, a_{4}, a_{5}$. The other is by putting $T_{M} x=x$ for every $x$. We have throughout the M-convexity theory assumed the minimum possible geometric condition of the existence of an intermediate point for every pair of points in a metric space. The results of Hardy and Rogers follow just by immitating our proof with the substitution $T_{M} x=x$ for every $x$, in which case the condition of commutativity of $T$ and $T_{M}$ becomes redundant just as the existence of an M-convexity point is. The results of Chatterjee [6] and Ghosh [7] also follow immediately from our theorem.

DEFINITION. A metric space $(X, d)$ is said to be M-convex if for every pair of points $x, y$ in $x$, there exists another point $z$ in $x$ such that $d(x, y)=d(x, z)+d(z, y)$. For strictly li-convex space and other related notions, the readers are referred to Chatterjee [8].

Let $(X, d)$ be an M-convex metric space. Then we can make the following definition. DEFINITION. A self-map $T$ on $X$ is said to be a generalized mean value contraction if, for every pair of points $x, y \in x$, there exist non-negative real numbers $a_{1}$, $a_{2}$, $a_{3}, a_{4}$ and $a_{5}$ such that

$$
\begin{aligned}
\mathrm{d}\left(\mathrm{TT}_{M} \mathrm{x}, \mathrm{TT}_{M} \mathrm{y}\right) \leq \mathrm{a}_{1} \mathrm{~d}(\mathrm{x}, \mathrm{y}) & +\mathrm{a}_{2} \mathrm{~d}\left(\mathrm{x}, \mathrm{TT}_{M} \mathrm{x}\right)+\mathrm{a}_{3} \mathrm{~d}\left(\mathrm{y}, \mathrm{TT}_{M} \mathrm{y}\right) \\
& +\mathrm{a}_{4} \mathrm{~d}\left(\mathrm{x}, \mathrm{TT}_{M} \mathrm{y}\right)+\mathrm{a}_{5} \mathrm{~d}\left(\mathrm{y}, \mathrm{TT}_{M} \mathrm{x}\right),
\end{aligned}
$$

where $\sum_{i=1}^{5} a_{i}<1$ and $T_{M} x$ is the point obtained from the M-convexity as follows

$$
d(x, T x)=d\left(x, T_{M} x\right)+d\left(T_{M} x, T x\right) \text {. }
$$

If the condition $\sum_{i=1}^{5} a_{i}<1$ is changed to $\sum_{i=1}^{5} a_{i}=1$ in the above, the said selfmap $\mathrm{T}$ will be called a generalized mean value non-expansive mapping.

Examples of such contractions and non-expansive mappings occur abundantly. We give one example. 
EXAMPLE. Let $X=\{x \in Q ; 0 \leq x \leq 1\}$ where $Q$ is the set of rational numbers and let $d(x, y)=|x-y|$. Then $(x, d)$ is an M-convex metric space. Now we define a selfmap $\mathrm{T}$ on $\mathrm{X}$ as follows

$$
\begin{aligned}
\mathrm{Tx} & =1-\mathrm{x} & \text { if } & 0 \leq \mathrm{x} \leq \frac{1}{2} \\
& =\mathrm{x}-\frac{1}{2} & \text { if } & \frac{1}{2} \leq \mathrm{x} \leq 1
\end{aligned}
$$

Then it is easy to prove, by taking $T_{M} x$ to define the usual midpoint of $x$ and $T x$, that $\mathrm{T}$ is a generalized mean value contraction (c.f. Wong [4]).

\section{MAIN THEOREMS.}

THEOREM 1. If $\mathbf{T}$ is a generalized mean value contraction from an M-convex complete metric space $(X, d)$ into itself and $T$ and $T_{M}$ commute on $X$, the derived set of $X$, then $\mathrm{T}$ has a unique fixed point.

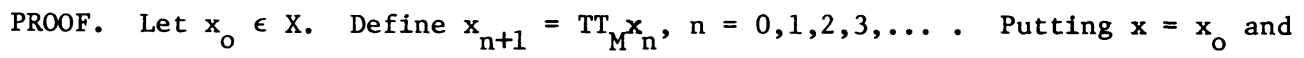
$y=x_{1}$ in $(1.1)$, we get

$$
\begin{aligned}
& d\left(x_{1}, x_{2}\right) \leq a_{1} d\left(x_{0}, x_{1}\right)+a_{2} d\left(x_{0}, x_{1}\right)+a_{3} d\left(x_{1}, x_{2}\right)+a_{4} d\left(x_{0}, x_{2}\right) \\
& \quad \text { for some }\left\{a_{i}\right\}_{i=1}^{4}, \sum_{i=1}^{4} a_{i}<1, a_{i} \geq 0,
\end{aligned}
$$

Again putting $x=x_{1}$ and $y=x_{0}$ in (1.1), we obtain

$$
\begin{aligned}
& d\left(x_{2}, x_{1}\right) \leq a_{1}^{\prime} d\left(x_{1}, x_{0}\right)+a_{2}^{\prime} d\left(x_{1}, x_{2}\right)+a_{3}^{\prime} d\left(x_{0}, x_{1}\right)+a_{5}^{\prime} d\left(x_{0}, x_{2}\right) \\
& \quad \text { for some }\left\{a_{i}^{\prime}\right\}, i=1,2,3,5, \sum_{\substack{i=1 \\
i=4}}^{5} a_{i}^{\prime}<1, a_{i}^{\prime} \geq 0,
\end{aligned}
$$

Adding (2.1) and (2.2), we now get

$$
\begin{array}{ll}
2 d\left(x_{1}, x_{2}\right) \leq\left(a_{1}+a_{2}+a_{1}^{\prime}+a_{3}^{\prime}\right) d\left(x_{0}, x_{1}\right)+\left(a_{2}^{\prime}+a_{3}\right) d\left(x_{1}, x_{2}\right)+\left(a_{4}+a_{5}^{\prime}\right) d\left(x_{0}, x_{2}\right) \\
\text { or }\left(2-a_{2}^{\prime}-a_{3}\right) d\left(x_{1}, x_{2}\right) \leq\left(a_{1}+a_{2}+a_{1}^{\prime}+a_{3}^{\prime}\right) d\left(x_{0}, x_{1}\right)+\left(a_{4}, a_{5}^{\prime}\right) d\left(x_{0}, x_{1}\right)+\left(a_{4}+a_{5}^{\prime}\right) d\left(x_{1}, x_{2}\right) \\
\text { or } \\
\text { Or } \\
\text { or } \left.2-a_{2}^{\prime}-a_{3}-a_{4}-a_{5}^{\prime}\right) d\left(x_{1}, x_{2}\right) \leq\left(a_{1}+a_{2}+a_{1}^{\prime}+a_{3}^{\prime}+a_{4}+a_{5}^{\prime}\right) d\left(x_{0}, x_{1}\right) \\
d\left(x_{1}, x_{2}\right) \leq \rho_{1} d\left(x_{0}, x_{1}\right) \text { where } \rho_{1}=\frac{a_{1}+a_{2}+a_{1}^{\prime}+a_{3}^{\prime}+a_{4}+a_{5}^{\prime}}{2-a_{2}^{\prime}-a_{3}-a_{4}-a_{5}^{\prime}} .
\end{array}
$$

By virtue of the conditions $\sum_{i=1}^{4} a_{i}<1$ and $\sum_{i=1}^{4} a_{i}^{\prime}<1$, it is easy to see that $0<\rho_{1}<1$.

By induction, we can prove that

$$
d\left(x_{n}, x_{n+1}\right) \leq \rho_{1}, \rho_{2}, \rho_{3}, \ldots, \rho_{n} d\left(x_{o}, x_{1}\right),
$$


where $0<\rho_{j}<1$ for $j=1,2, \ldots, n$.

So $\left\{x_{n}\right\}$ is a Cauchy sequence and therefore by completeness $\left\{x_{n}\right\}$ converges to, say $\zeta$.

Then

$$
\begin{aligned}
& d\left(\zeta, \operatorname{TT}_{M}\right) \leq d\left(\zeta, x_{n+1}\right)+d\left(x_{n+1}, \operatorname{TT}_{M^{\beta}}\right) \\
& \leq d\left(\zeta, x_{n+1}\right)+d\left(T_{M} x_{n}, T_{M} \zeta\right) \\
& \leq d\left(\zeta, x_{n+1}\right)+a_{1} d\left(x_{n}, \zeta\right)+a_{2} d\left(x_{n}, T T_{M} x_{n}\right) \\
& +a_{3} d\left(\zeta, T T_{M}\right)+a_{4} d\left(x_{n}, T T_{1} \zeta\right)+a_{5} d\left(\zeta, T_{M} x_{n}\right) . \\
& \leq d\left(\zeta, x_{n+1}\right)+a_{1}^{\prime \prime} d\left(x_{n}, \zeta\right)+a_{2}^{\prime \prime} d\left(x_{n}, x_{n+1}\right) \\
& +a_{3}^{\prime \prime} d\left(\zeta, \operatorname{TT}_{N} \zeta\right)+a_{4}^{\prime \prime} d\left(x_{n}, \operatorname{TT}_{N} \zeta\right)+a_{5}^{\prime \prime} d\left(\zeta, x_{n+1}\right) . \\
& \leq\left(a_{3}^{\prime \prime}+a_{4}^{\prime \prime}\right) d\left(\zeta, T_{M} \zeta\right) \text { as } n \rightarrow \infty \text {. }
\end{aligned}
$$

Thus $\mathrm{d}\left(\zeta, \mathrm{TT}_{\mathrm{M}} \zeta\right)=0$.

This implies $\mathrm{TT}_{M^{\zeta}}=\zeta$.

Now, we proceed to prove that $\zeta$ is the fixed point as desired.

$$
\begin{aligned}
& \mathrm{d}(\zeta, T \zeta)=\mathrm{d}\left(\operatorname{TT}_{\mathbb{N}^{\zeta}}, \mathrm{T}\left(\mathrm{TT}_{\mathrm{N}^{\beta}}\right)\right) \\
& =\mathrm{d}\left(\mathrm{TT}_{\mathrm{M}^{\zeta}}, \mathrm{T}\left(\mathrm{T}_{\mathrm{M}} \mathrm{T \zeta}\right)\right) \quad \text { [since } \mathrm{TT}_{\mathrm{M}^{\zeta}}=\mathrm{T}_{\mathrm{M}} \mathrm{T \zeta}^{\zeta} \text { for } \zeta \in \mathrm{X}^{\prime} \text { ] } \\
& =\mathrm{d}\left(\mathrm{TT}_{\mathrm{M}}, \mathrm{TT}_{\mathrm{M}}(\mathrm{T \zeta})\right) \\
& \leq a_{1}^{\prime \prime \prime} d(\zeta, T \zeta)+a_{2}^{\prime \prime \prime} d\left(\zeta, T_{M}(T \zeta)\right)+a_{3}^{\prime \prime \prime} d\left(T \zeta, T_{M P}\right) \\
& +a_{4}^{\prime \prime \prime} d\left(\zeta, T_{M}(T \zeta)\right)+a_{5}^{\prime \prime \prime} d\left(T \zeta, T T_{M}\right) \text {. } \\
& \leq a_{1}^{\prime \prime} d(\zeta, T \zeta)+a_{2}^{\prime \prime \prime} d(\zeta, T \zeta)+a_{3}^{\prime \prime \prime} d(\zeta, T \zeta) \\
& +a_{4}^{\prime \prime \prime} d(\zeta, T \zeta)+a_{5}^{\prime \prime \prime} d(\zeta, T \zeta) \text {. } \\
& d(\zeta, T \zeta) \leq 0 \text { since } \sum_{i=1}^{5} a_{i}^{\prime \prime \prime}<1 \text {. }
\end{aligned}
$$

Thus $d(\zeta, T \zeta)=0$ which implies $T \zeta=\zeta$; i.e. $\zeta$ is a fixed point. The uniqueness is easy to verify.

This completes the proof.

REMARK. One should note that in the above theorem, we have used completeness only in getting the limit $\zeta$ and we have used the commutativity of $T$ and $T_{M}$ only for $\zeta$. So we have actually proved the following more general theorem. 
THEOREM 2. If $T$ is a generalized mean value contraction from an $M$-convex metric space $(X, d)$ into itself and there exists a point $x_{0} \in X$ such that

(i) $x_{n}$ converges to a point $\zeta$ when $x_{n}=T T_{M} x_{n+1}$ and

(ii) $\mathrm{TT}_{\mathrm{M}} \zeta=\mathrm{T}_{\mathrm{M}} \mathrm{T}$,

then $T$ has, a unique fixed point. The following theorem now follows from the above theorem.

THEOREM 3. Let $(\mathrm{X}, \mathrm{d})$ be a strictly M-convex compact metric space and $\mathrm{T}$ be a generalized mean value non-expansive mapping from $X$ into itself such that $T$ and $T_{M}$ commutes on $\mathrm{X}$, then $\mathrm{T}$ has a fixed point.

PROOF. Define $f(x)=d(x, T x)$.

Then the continuity of $d$ gives the continuity of $f$. Now $x$ being compact, $f$ attains its minimum at a point, say $x_{0} \in X$.

$$
\begin{aligned}
& \text { If } d\left(T x_{0}, x_{0}\right)=0 \text {, then } x_{0} \text { is the fixed point. } \\
& \text { So suppose } \mathrm{Tx} \mathrm{x}_{0}=\mathrm{x}_{0}, \text { i.e. } \mathrm{d}\left(\mathrm{x}_{0}, \mathrm{Tx_{0 } )}>0\right. \text {. } \\
& \text { Then } f\left(T_{M} x_{0}\right)=d\left(T_{M} x_{0}, T\left(T_{M} x_{0}\right)\right) \\
& =d\left(T_{M} x_{0}, T_{M}\left(T x_{0}\right)\right) \\
& <\mathrm{d}\left(\mathrm{x}_{\mathrm{o}}, \mathrm{Tx_{0 }}\right) \text {, by strict M-convexity and Theorem } 2 \text {. } \\
& =f\left(x_{0}\right) \text {, a contradiction. }
\end{aligned}
$$

Hence $T_{x} 0=x_{0}$.

REMARR 1. We can drop the condition of strict M-convexity if we take "a generalized mean value contraction" in place of "non-expansive mapping".

REMARK 2. Our method of proof in the above theorems is a substantial modification and fusion of the methods due to Hardy and Rogers [5] and Wong [4].

3. APPLICATIONS.

Since the inception, the fixed point notions have found many glorious applications in the fields of Economic Stability Theory, the Theory of Differential Equations, Control Theory, the Theory of Integral Equations, and Fluid Dynamics. The existence problems which are fundamental in mathematics, have often been solved by the fixed point theorems. We feel that our theorems can be applied to more general situations than eyer, since ours is applicable to F-spaces, Frechet spaces, and, more general1y, to 
metric spaces with the minimum geometric structure. We give below a nice application in a situation where the basic space is not linear, but a metric space with the minimum geometric structure.

EXAMPLE. Let $X=\left\{e^{i \theta}, 0 \leq \theta<2 \pi\right\}$. Then $X$ is a complete M-convex metric space where the metric $d$ is defined as $d\left(e^{i \theta_{1}}, e^{i \theta_{2}}\right)=\left|\theta_{1}-\theta_{2}\right|$. We take, in particular, $T_{M}$ to represent the midpoint of the arc between $e^{i \theta}$ and $T\left(e^{i \theta}\right)$ where $T\left(e^{i \theta}\right)$ is defined as $T\left(e^{i \theta}\right)=e^{i \theta / 2}$. Here $i t$ is easy to show that $T$ satisfies all the conditions of our Theorem 1 and hence the existence of a fixed point of $T$ is guaranteed. In fact, the point $e^{i 0}$, i.e. 1 , is the fixed point.

ACKNOWLEDGEMENT. The author finds it a great pleasure to thank the referee for his suggestion for substantial improvement of the paper. Thanks are due to Dr. M. K. Das without whose constant inspiration this paper could not be completed.

\section{REFERENCES}

1. EDELSTEIN, M. On Fixed and Periodic points under contractive mappings, Pacific J. Math. 37 (1962) 74-79.

2. KANNAN, R. Some Results on Fixed Points, Bull. Cal. Math. Soc. 60 (1968) 71-76.

3. REICH, S. Fixed Points of Contractive Functions, Bul1. Un. Math. Ital. 4 (1972) $26-42$.

4. WONG, C.S. Fixed Points and Characterizations of Certain Maps, Pacific J. Math. 54 (1974) 305-312.

5. HARDY, G. and ROGERS, T. A Generalization of a Fixed Point Theorem of Reich, Canad. Math. Bul1. 16 (1973) 201-206.

6. CHATTERJEA, S.K. Fixed Point Theorems, C.R. Acad. Bulgare Sci. 25 (1972) 727-730.

7. GHOSH, K.M. A Generalization of Contraction Principle, Int. J. Math. and Math. Sci. 4 (1981) 201-206.

8. CHATTERJEE, D. M-Convexity and Best Approximations, Pub. Inst. Math. $\underline{28}(42)$, (1980) 43-50. 


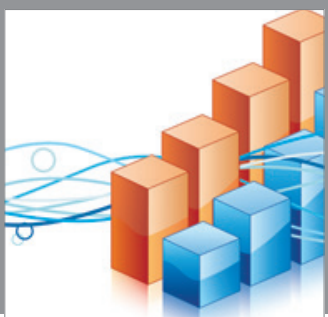

Advances in

Operations Research

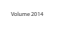

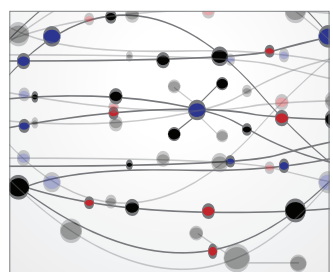

\section{The Scientific} World Journal
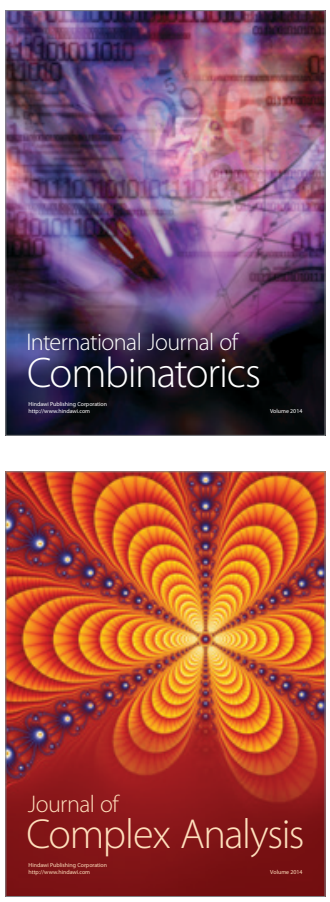

International Journal of

Mathematics and

Mathematical

Sciences
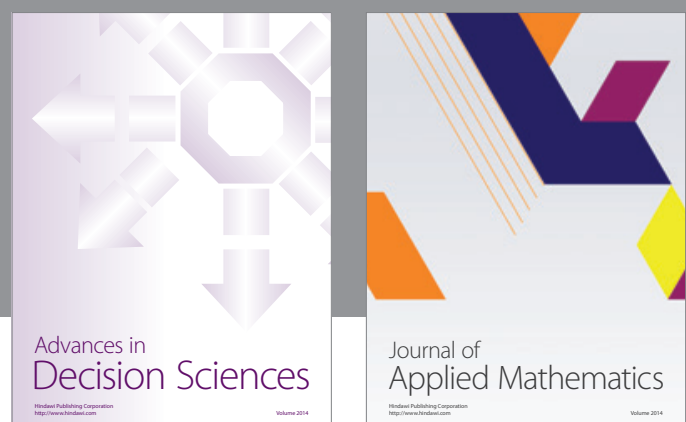

Journal of

Applied Mathematics
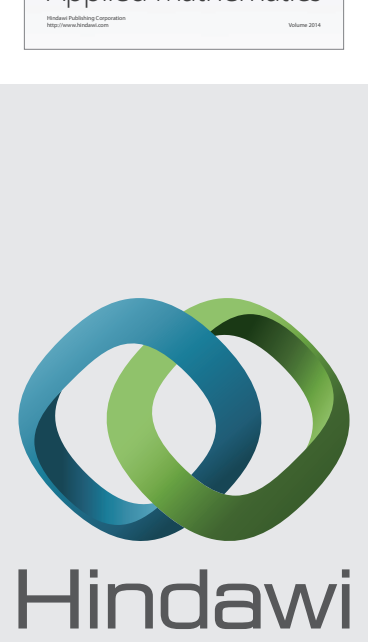

Submit your manuscripts at http://www.hindawi.com
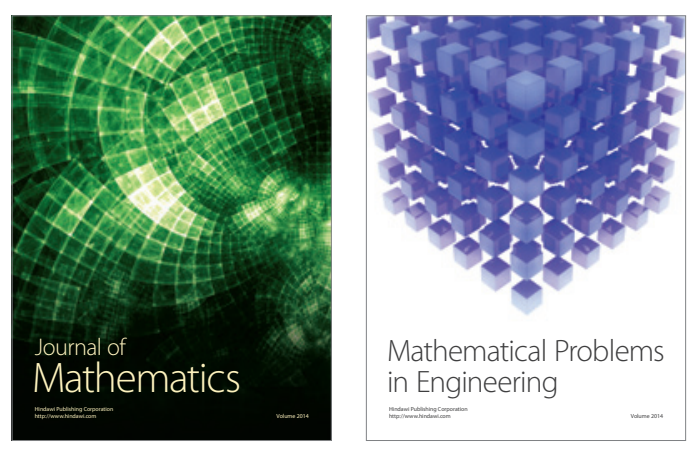

Mathematical Problems in Engineering
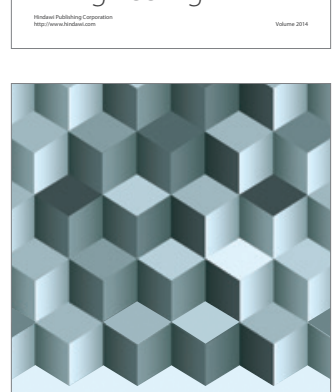

Journal of

Function Spaces
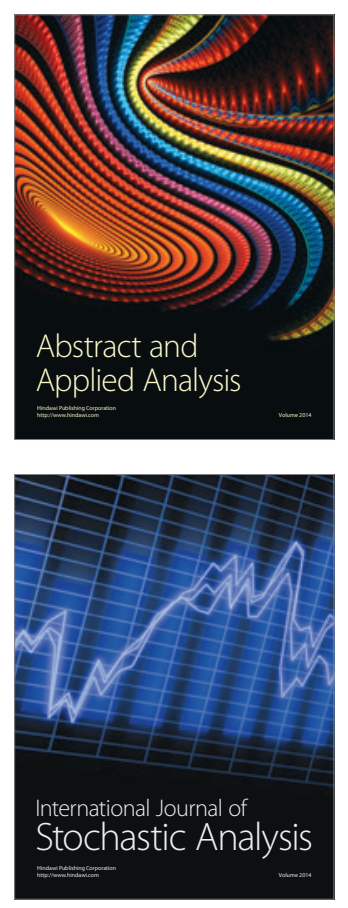

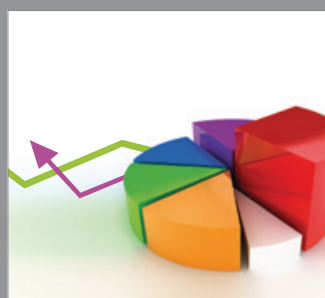

ournal of

Probability and Statistics

Promensencen
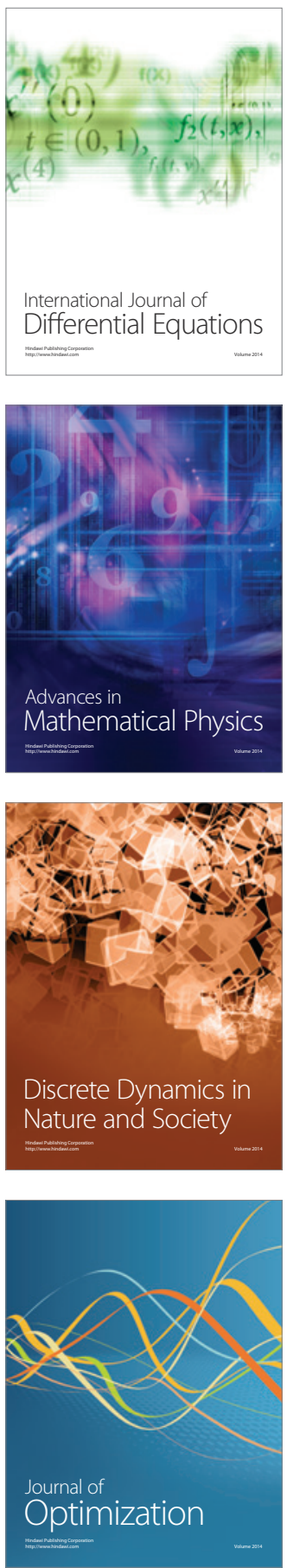